\title{
High-Efficiency Electromagnetic Wave Controlling with All-Dielectric Huygens' Metasurfaces
}

\author{
Zheng-bin Wang, ${ }^{1}$ Jin Shi, ${ }^{2}$ and Jin-chang Chen ${ }^{1}$ \\ ${ }^{1}$ College of Electronic Science and Engineering, Nanjing University of Posts and Telecommunications, Nanjing 210023, China \\ ${ }^{2}$ School of Electronics and Information, Nantong University, Nantong 226019, China \\ Correspondence should be addressed to Zheng-bin Wang; wangzb@njupt.edu.cn
}

Received 13 February 2015; Revised 5 May 2015; Accepted 6 May 2015

Academic Editor: Paolo Burghignoli

Copyright (c) 2015 Zheng-bin Wang et al. This is an open access article distributed under the Creative Commons Attribution License, which permits unrestricted use, distribution, and reproduction in any medium, provided the original work is properly cited.

\begin{abstract}
Subwavelength dielectric blocks with varying thicknesses are introduced to realize $0 \sim 2 \pi$ phase change. A Huygens' metasurface composed of such nonuniform building blocks is shown to efficiently refract normally incident waves in a broadband. According to the same physical mechanism, we fabricate an electrically thin lens with concentric subwavelength dielectric blocks and integrate it with a patch antenna to form a three-dimensional (3D) ultra-low-profile lens antenna system. Full-wave simulation demonstrates the lens antenna's excellent performances in high directivity, broadband, low loss, and low side-lobe levels.
\end{abstract}

\section{Introduction}

In recent years, the development of metamaterials has opened up the researcher's minds on how to control electromagnetic (EM) waves from microwave to optical frequencies $[1,2]$, or even acoustic waves [3,4]. Most EM metamaterials are fabricated by arranging a set of subwavelength scatters throughout a region of space, which can generate the required EM properties as bulk materials [5]. More recently, the concept was further extended by wisely placing electrically small structures into a planar pattern [6-12]. These microscopic scatterers can modulate the phase and amplitude of each Huygens' secondary source on the interface and in turn manipulate the transmitted EM waves. Such electrically thin interfaces are called metafilms, or more generally, metasurfaces. In the past few years, most of the reported metasurface designs relied on resonant anisotropic elements, such as V-shaped antennas [6, 7], H-shaped antennas [8,9], and dipoles [10]. Such approaches are narrowbanded, sensitive to electric polarization, and the transmission efficiency is also very low (on the order of $5 \%$ ), which restrict them from further applications. Last year, Pfeiffer et al. presented a novel metasurface that can bend light efficiently by cascading three patterned, metallic sheets [11]. The transmission efficiency can be up to $30 \%$ at a specific frequency.
In this work, we present another high-efficiency approach to Huygens' metasurfaces, which are composed of alldielectric electrically thin blocks. Such metasurfaces are broadbanded, easy to fabricate, high efficient, and insensitive to electric polarization. In addition, compared with metallic approaches, all-dielectric metasurfaces are immune to some negative effects brought by high-power incident EM waves, such as heating due to ohmic losses, arcing at field concentration points. A refractive metasurface is first proposed to demonstrate the feasibility of the all-dielectric approach. Then, an all-dielectric metasurface lens is designed to focus incident plane waves. Finally, the lens is further integrated with a patch antenna to form a three-dimensional (3D) ultralow-profile lens antenna system, which exhibits high directivity, broadband, low side-lobe levels, and steerable capabilities.

\section{A Huygens' Metasurface Composed of Electrically Thin Dielectric Building Blocks}

Figure 1 shows a typical subwavelength dielectric building block of the metasurface. The length and height of the square unit cell are $l$ and $h$, respectively. The relative permittivity of the dielectric substrate is $\varepsilon_{r}$. Now consider an incident EM 


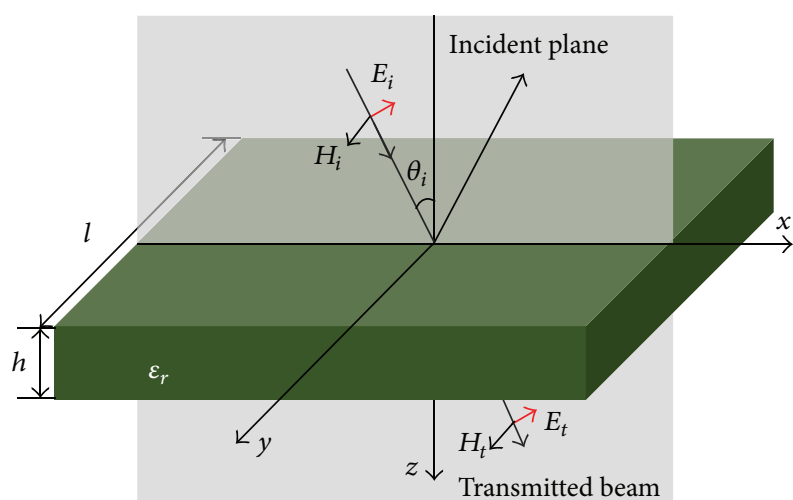

(a)

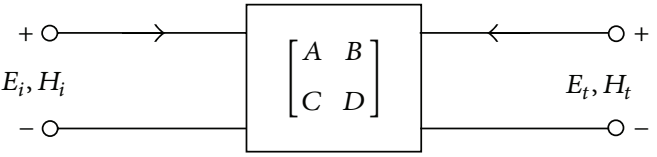

(b)

FIGURE 1: (a) 3D topology of a subwavelength dielectric building block for oblique incident TM waves and (b) the equivalent two-port transmission line circuit model.

beam with transverse magnetic (TM) polarization transmitting through the element. The incident plane is $x z_{\perp}$ plane of the local coordinate system, and the incident angle is $\theta_{i}$. Assume each dielectric block as a homogeneous planar layer; a two-port network model (shown in Figure 1(b)) can be obtained according to the transmission line theory $[12,13]$. The TM fields on the surfaces $z=0$ and $z=h$ can be related to each other through the $A B C D$ matrix as shown as follows:

$$
\begin{aligned}
& {\left[\begin{array}{l}
E_{x}(z) \\
H_{y}(z)
\end{array}\right]_{z=0}} \\
& \quad=\left[\begin{array}{cc}
\cos (\beta h) & j Z_{c} \sin (\beta h) \\
j Y_{c} \sin (\beta h) & \cos (\beta h)
\end{array}\right]\left[\begin{array}{c}
E_{x}(z) \\
H_{y}(z)
\end{array}\right]_{z=h},
\end{aligned}
$$

where $\beta=\omega \sqrt{\left(\varepsilon_{r}-\sin ^{2} \theta_{i}\right) \varepsilon_{0} \mu_{0}}, Z_{c}=1 / Y_{c}=\eta_{0} / \sqrt{\varepsilon_{r}-\sin ^{2} \theta_{i}}$. $\omega$ and $\eta_{0}$ are the angular frequency and the characteristic impedance of free space, respectively. The tangential fields at the top $(z=0)$ and bottom $(z=h)$ surfaces are the same, which leads to the boundary conditions

$$
\begin{aligned}
E_{x}(0) & =-\eta_{0} \cos \theta_{i} H_{y}(0)+2 E_{i} \cos \theta_{i}, \\
\frac{E_{x}(h)}{H_{y}(h)} & =\eta_{0} \cos \theta_{i} .
\end{aligned}
$$

Combine (1) and (2); we can obtain the transmission coefficient of the element

$$
\begin{aligned}
& T_{\mathrm{TM}} \\
& \quad=\frac{E_{x}(h)}{E_{i}} \\
& \quad=\frac{2 \eta_{0} \cos ^{2} \theta_{i}}{2 \eta_{0} \cos (\beta h) \cos \theta_{i}+j \sin (\beta h)\left(Z_{c}+\eta_{0}^{2} Y_{c} \cos ^{2} \theta_{i}\right)},
\end{aligned}
$$

which presents the exact transmission field on the bottom surface of the dielectric block. According to the duality theorem, the transmission coefficient of transverse electric (TE) polarization waves can also be obtained

$$
\begin{aligned}
& T_{\mathrm{TE}} \\
& \quad=\frac{E_{y}(h)}{E_{i}} \\
& =\frac{2 \eta_{0} \cos ^{2} \theta_{i}}{2 \eta_{0} \cos (\beta h) \cos \theta_{i}+j \sin (\beta h)\left(\eta_{0}^{2} Y_{c}+Z_{c} \cos ^{2} \theta_{i}\right)} .
\end{aligned}
$$

Through modeling the electric field on the surface as an aperture field distribution, we can further obtain the far-field pattern.

From (3) we can extract the phase shift of the transmitted field that has propagated through the dielectric layer

$$
\varphi_{\mathrm{TM}}(T)=\tan ^{-1}\left[\frac{Z_{c}+Y_{c} \eta_{0}^{2} \cos ^{2} \theta_{i}}{2 \eta_{0} \cos \theta_{i}} \tan \beta h\right] .
$$

The phase shift of the transmitted TE waves can be extracted from (4)

$$
\varphi_{\mathrm{TE}}(T)=\tan ^{-1}\left[\frac{Y_{c} \eta_{0}^{2}+Z_{c} \cos ^{2} \theta_{i}}{2 \eta_{0} \cos \theta_{i}} \tan \beta h\right] .
$$

From the above equations we can see that if the incident angle $\left(\theta_{i}\right)$ and the dielectric constant $\left(\varepsilon_{r}\right)$ are given, the phase shift of the transmission field depends on the height of the building block, $h$. Here, we should notice that when the height of the dielectric block varies from $0 \sim \lambda_{0} / \sqrt{\varepsilon_{r}}$, the phase shift of the transmission field will change from 0 to $2 \pi$, which is essential for fully controlling the transmitted beam. When high-permittivity dielectrics are chosen, the total height of the metasurface will be far less than the freespace wavelength.

Then, a proof-of-concept Huygens' metasurface is designed to refract normally incident plane waves $\left(\theta_{i}=0\right)$ in a wide band. Figure 2(a) shows the super unit cell of an all-dielectric nonuniform metasurface, which is composed of eight subwavelength square blocks with different heights 

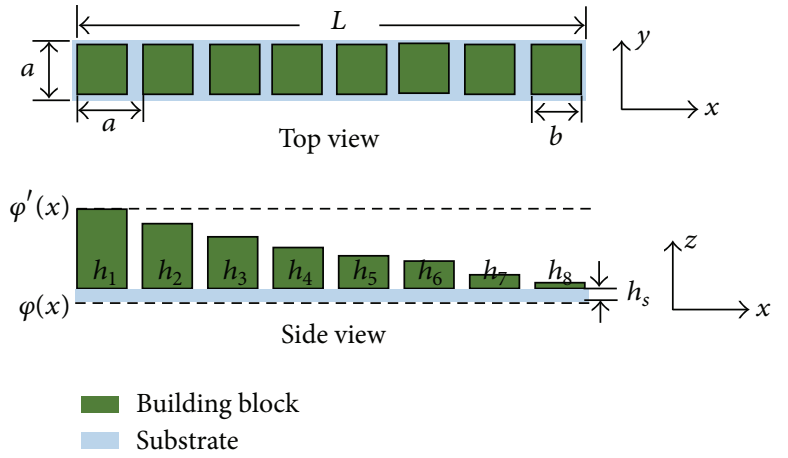

(a)

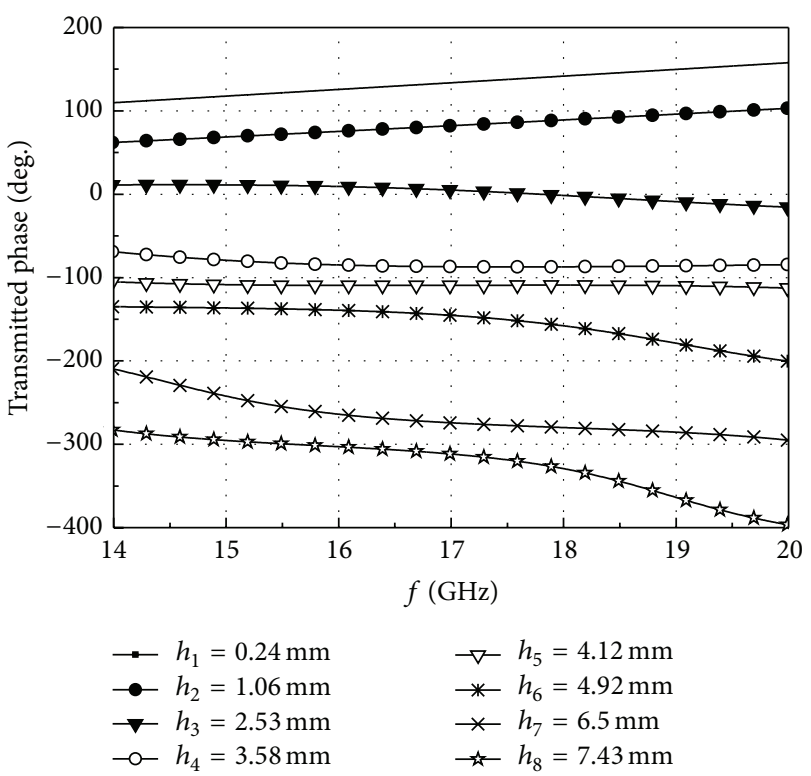

(b)

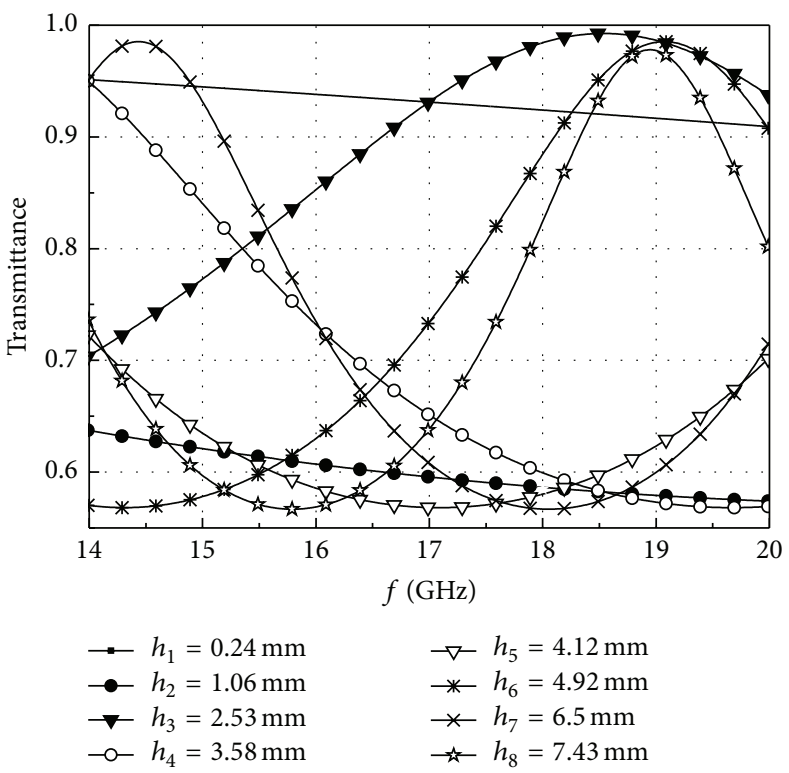

(c)

Figure 2: (a) Top and side view of the super unit cell of an electrically thin metasurface, which is composed of eight RO3210 dielectric building blocks and a RT5880LZ substrate. The detailed geometry of the super unit cell, in which $a=6.25 \mathrm{~mm}, b=5.5 \mathrm{~mm}, h_{s}=0.76 \mathrm{~mm}$, $h_{1}=7.43 \mathrm{~mm}, h_{2}=6.50 \mathrm{~mm}, h_{3}=4.92 \mathrm{~mm}, h_{4}=4.12 \mathrm{~mm}, h_{5}=3.58 \mathrm{~mm}, h_{6}=2.53 \mathrm{~mm}, h_{7}=1.06 \mathrm{~mm}$, and $h_{8}=0.24 \mathrm{~mm}$; (b) the calculated frequency dependence of the transmitted phase shift, and (c) transmittance of each building block.

$\left(h_{1} \sim h_{8}\right)$ and a thin substrate with height, $h_{s}$. The width of each building block is $b$, which depends on the requirement for the gradient of phase discontinuity along the $x$ direction $(d \varphi / d x)[6,8]$. The dashed lines in Figure 2(a) refer to the incident phase distribution ( $\varphi_{\text {in }}(x)$ on the bottom plane) and the desired outgoing phase distribution $\left(\varphi_{\text {out }}(x)\right.$ on the top plane), respectively. If the distance between the two planes is far less than the operating wavelength in the free space, that is, electrically thin, the nonuniform dielectric structure still can be looked on as a surface. Here, Rogers board with high permittivity $\left(\mathrm{RO} 3210, \varepsilon_{r}=10.2, \tan \delta=0.0027\right)$ is chosen to fabricate the square blocks. As for the substrate, low-permittivity Rogers board is adopted (RT5880LZ, $\varepsilon_{r}=$ $1.96, \tan \delta=0.0019$ ). According to (5) and (6), the heights of the eight building blocks are optimized to make the interval of phase shift to be $\pi / 4$ around in a wide band, as shown in Figure 2(b). The frequency dependence of the transmittance of the eight blocks is shown in Figure 2(c). Obviously, the transmission efficiency of each block is above $55 \%$, which, to the best of our knowledge, is much higher than the reported 


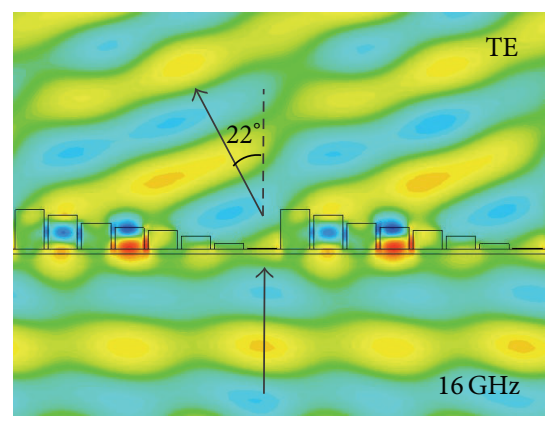

(a)

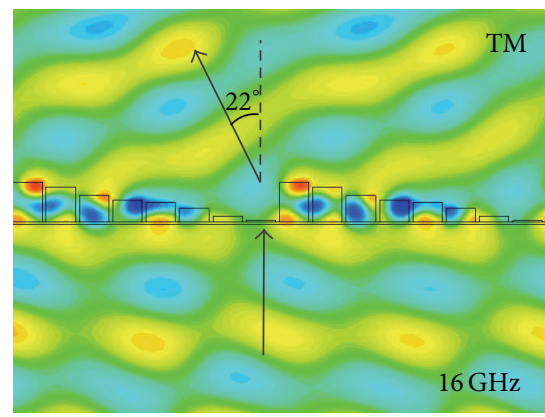

(d)

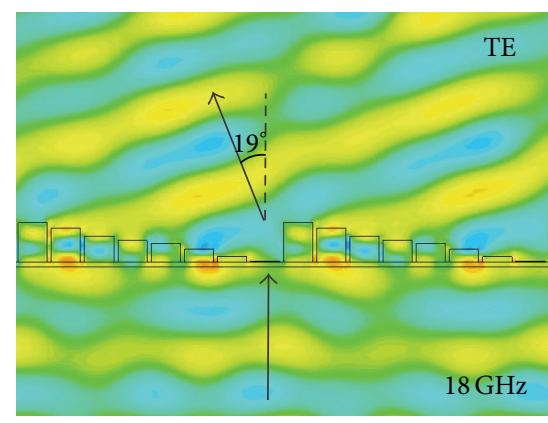

(b)

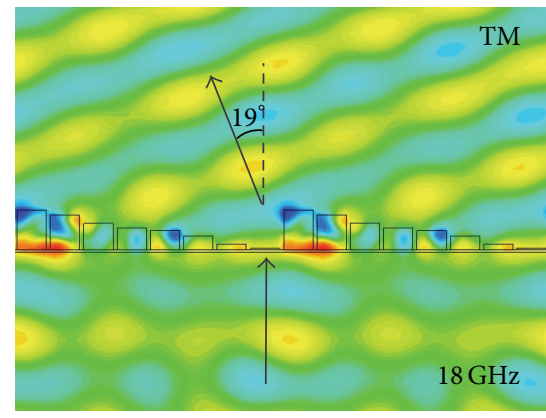

(e)

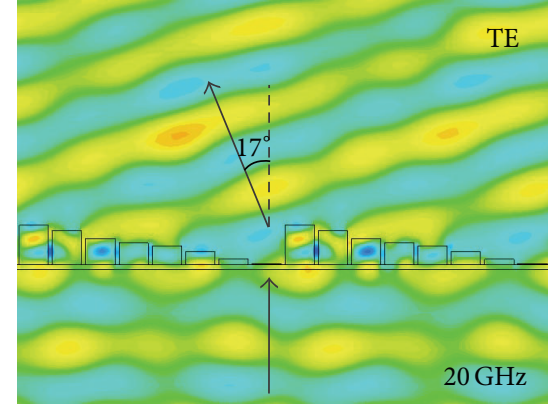

(c)

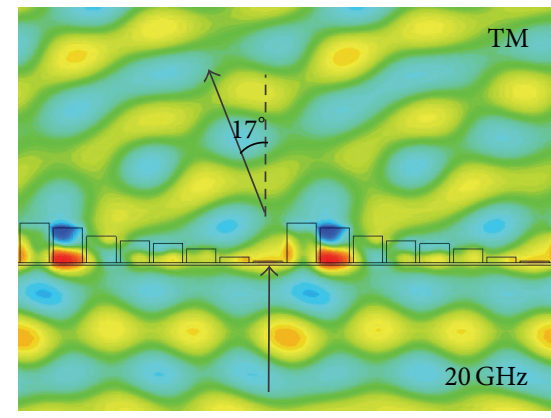

(f)

FIGURE 3: Time snapshots of the simulated steady-state electric field when plane waves are normally impinging on the nonuniform dielectric metasurface from the bottom. TE polarization incident waves (a) at $16 \mathrm{GHz}$, (b) at $18 \mathrm{GHz}$, and (c) at $20 \mathrm{GHz}$. TM polarization incident waves (d) at $16 \mathrm{GHz}$, (e) at $18 \mathrm{GHz}$, and (f) at $20 \mathrm{GHz}$.

metasurface designs [6-11]. Here, it should be noted that the transmittances of each block are not identical, which will bring a little aberration in the transmitted field. In addition, the nonuniformity of the refract-array's surface will also introduce some aberration.

By extending the super unit cell in both $x$ and $y$ direction we can fabricate a refractive metasurface. Its gradient of phase discontinuity along $x$ direction is $d \varphi / d x=2 \pi / L$ ( $L$ is the length of the super unit cell). Then, the corresponding refractive angle can be obtained from the general Snell's law [6]

$$
\theta_{t}=\sin ^{-1}\left(\frac{\lambda_{0}}{L}\right)
$$

where $\lambda_{0}$ is the free-space wavelength of the incident wave. Figure 3 shows the time snapshots of the steady-state electric field simulated in the Computer Simulation Technology (CST) Microwave Studio, which is based on the finite integration method [14]. Plane waves with TE (Figures 3(a)-3(c)) and TM (Figures 3(d)-3(f)) polarization electric fields are normally incident at the metasurface. From these figures we can see that the nonuniform metasurface refracts the incident waves effectively in a wide band $(16 \sim 20 \mathrm{GHz})$. And the refractive angle decreases with the operating frequency, which is consistent with the theoretical model in (6). Therefore, Huygens' metasurfaces based on electrically thin dielectric blocks can be used to fully control transmitted electromagnetic waves and in turn to fabricate high-efficiency electromagnetic lenses.

\section{Ultra-Low-Profile Lens Antenna Design and Analysis}

In this section, the ability of the high-efficiency metasurface's fully controlling transmitted waves is utilized to fabricate a $3 \mathrm{D}$ ultra-low-profile lens antenna system. As we all know, lens antenna systems have been intensively studied throughout the microwave, millimeter-wave, and optical bands for their special property of radiating point-source EM waves at the focal point with high directivities, such as parabolic lens antennas [15], Fresnel lens antennas [16], and elliptic lens antennas [17]. Traditional dielectric lenses modulate applied waves by gradual phase changing, which is realized by engineering the curve surface topography or the refractive index of the spatial profile [18-21]. However, the lens with curve surface is bulky and heavy at low frequencies, and with the closing of the focal point to the lens surface, the total volume of the antenna system will be too large to be acceptable. As for the planar lens with varying refractive index, it is still very hard and complicated to fabricate it now.

Here, we propose an ultra-low-profile lens antenna operated at $\mathrm{Ku}$ band, which is composed of an electrically thin dielectric metasurface lens and a planar patch antenna. Figure 4 shows the detailed schematic of the antenna system. The metasurface lens is composed of many sector dielectric blocks, which can be clapped with Rogers' RO3210 boards. The height of each building block can be obtained from (5) or (6), where the phase distribution at the radial direction 


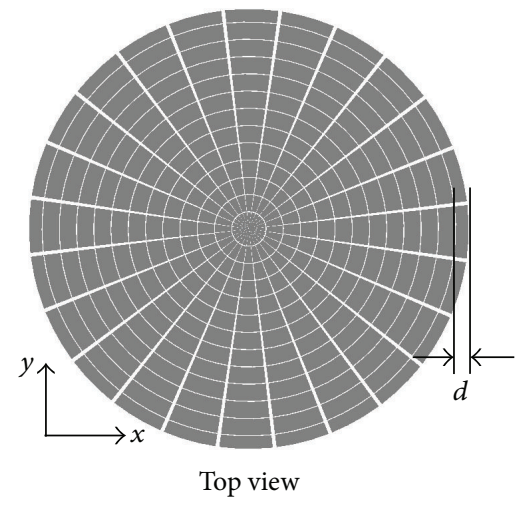

(a)

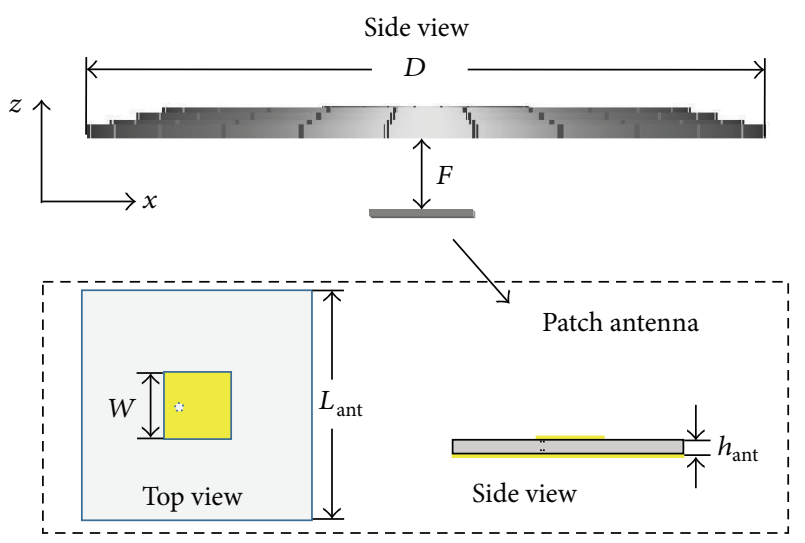

(b)

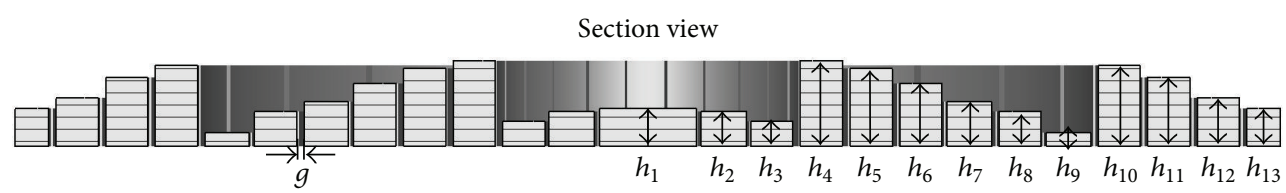

(c)

FiguRE 4: The detailed geometry of the ultra-low-profile lens antenna system, which is composed of a clapped dielectric metasurface and a patch antenna. The geometry of the lens: $d=4 \mathrm{~mm}, h_{1}=3.05 \mathrm{~mm}, h_{2}=2.78 \mathrm{~mm}, h_{3}=2.00 \mathrm{~mm}, h_{4}=6.84 \mathrm{~mm}, h_{5}=6.20 \mathrm{~mm}, h_{6}=5.04 \mathrm{~mm}$, $h_{7}=3.56 \mathrm{~mm}, h_{8}=2.76 \mathrm{~mm}, h_{9}=1.11 \mathrm{~mm}, h_{10}=6.45 \mathrm{~mm}, h_{11}=5.50 \mathrm{~mm}, h_{12}=3.84 \mathrm{~mm}, h_{13}=3.00 \mathrm{~mm}$, and $g=0.2 \mathrm{~mm}$. The focal length and the diameter of the lens are $F=15 \mathrm{~mm}$ and $D=102 \mathrm{~mm}$, respectively. The geometry of the antenna: $w=4.6 \mathrm{~mm}, L_{\text {ant }}=17 \mathrm{~mm}$, and $h_{\text {ant }}$ $=0.813 \mathrm{~mm}$.

depends on the equal optical path principle of convex lens [22]

$$
\varphi(r)=2 N \pi+k_{0}\left(\sqrt{r^{2}+F^{2}}-F\right),
$$

where $N$ is an arbitrary integer and $r$ and $F$ are the radial distance and the focal length, respectively. Here, the focal point should be designed as close as possible to the lens surface. Because of the mutual coupling between the lens and the patch antenna, as well as the need to make full use of the lens aperture, the focal length is optimized to be $F=15 \mathrm{~mm}$ $\left(\approx 3 \lambda_{0} / 4\right.$ of the centre frequency, $\left.f_{0}=15.5 \mathrm{GHz}\right)$. The width of each concentric sector block is $d=4 \mathrm{~mm}\left(\approx \lambda_{0} / 5\right)$, and the gap between them is $g=0.2 \mathrm{~mm}$. The total aperture size is $D$ $=102 \mathrm{~mm}\left(\approx 5.2 \lambda_{0}\right)$. The ratio of the lens's focal length to its aperture diameter is $F / D=0.15$ and can be further decreased by increasing the lens's diameter. A planar back-feed patch antenna acts as the primary feed and is located at the focal point. The radiating metal plate, measuring $W \times W=4.6 \mathrm{~mm}$ $\times 4.6 \mathrm{~mm}$, is etched on a square Rogers' RO4003 board $\left(\varepsilon_{r}=\right.$ $3.55, \tan \delta=0.0027, L_{\text {ant }} \times L_{\text {ant }}=17 \mathrm{~mm} \times 17 \mathrm{~mm}$ ). The height of the substrate is $h_{\text {ant }}=0.813 \mathrm{~mm}$.

As for the dielectric lens itself, it can converge the incident plane waves into the focal point very well. In Figure 5(a), an $x$-polarized plane wave (operated at $16 \mathrm{GHz}$ ) impinges on the metasurface lens from the $z$-direction with the amplitude of $1 \mathrm{v} / \mathrm{m}$. The simulated steady-state electric field $E_{x}$ shows a clear focal point in the $x-z$ plane. The measured focal length is $F=16.8 \mathrm{~mm}$. Figure $5(\mathrm{~b})$ shows the power distribution along the $x$-direction in the focal plane (at $z=16.8 \mathrm{~mm}$ ). Obviously, the transmitted power concentrates inside the main lobe, and the full width at half maximum (FWHM) is $12 \mathrm{~mm}(\sim 0.63 \lambda)$. Plane waves operated at other frequencies, such as $14.5 \mathrm{GHz}$ and $16.5 \mathrm{GHz}$, are also simulated (not shown here). These simulation results demonstrate that with the increasing of the operating frequency, both the focal length and the FWHM are slightly decreasing.

Consider that the radiating metal plate of the patch antenna is smaller than the focal spot; it can be looked on as the point source and the phase center. Figure 6(a) shows the simulated steady-state electric field of the lens antenna system at $16 \mathrm{GHz}$. Obviously, the metasurface lens transforms the spherical wavefront of the source antenna into a radiated plane wavefront. Figures $6(\mathrm{~b})-6(\mathrm{~d})$ show the simulated far-field gain of the patch antenna itself (the dashed line) and the integrated low-profile lens antenna (the solid line) at $14.5 \mathrm{GHz}, 15.5 \mathrm{GHz}$, and $16.5 \mathrm{GHz}$, respectively. From these figures we can see that the existence of the electrically thin metasurface lens makes the far-field gain of the patch antenna increase more than $10 \mathrm{~dB}$ and the 3 - $\mathrm{dB}$ angular width reduce to $15^{\circ}$, which means the directivity of the antenna is highly improved. These figures also demonstrate that the lens antenna system has more stable far-field gain (16.3-17.5 dB) in a wide band (14.5-16.5 GHz), and the side-lobe level is below $-13 \mathrm{~dB}$.

It should be noted that, since the spacing between the patch antenna and the lens is less than one wavelength, the mutual coupling effects have to be taken into consideration. This interesting issue will be discussed in detail in the future work. 


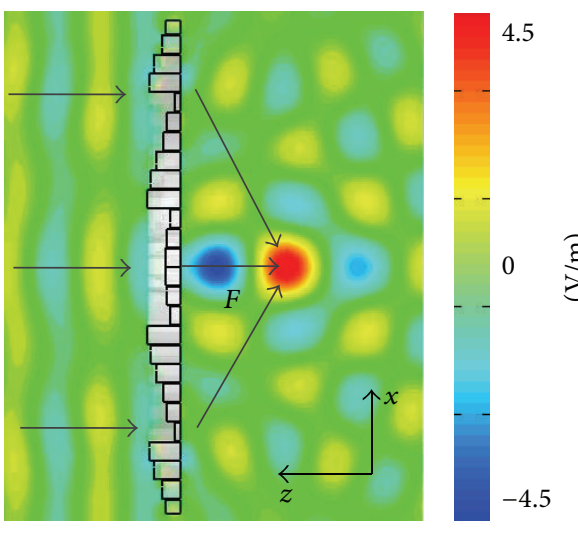

(a)

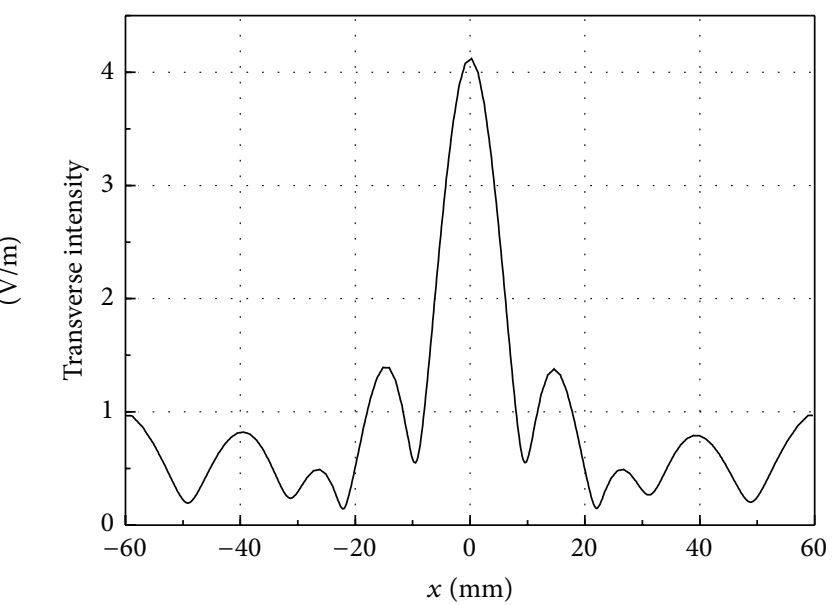

(b)

Figure 5: (a) The time snapshot of the steady-state electric field in the $x$ - $z$ plane; (b) intensity distribution of the steady-state electric field along the $x$-axis in the focal plane. The operating frequency of the incident plane waves is $16 \mathrm{GHz}$.

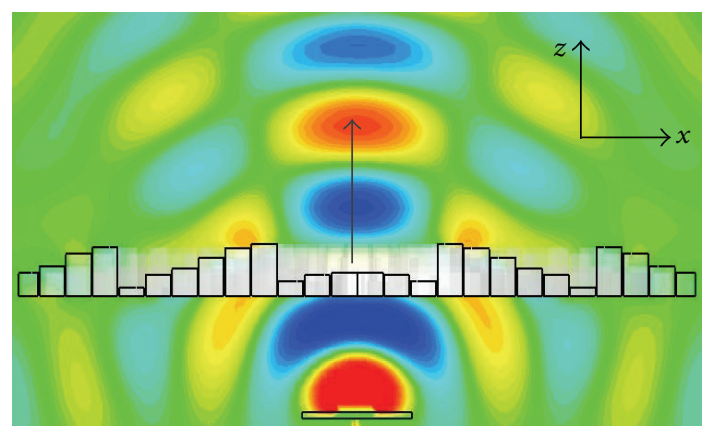

(a)

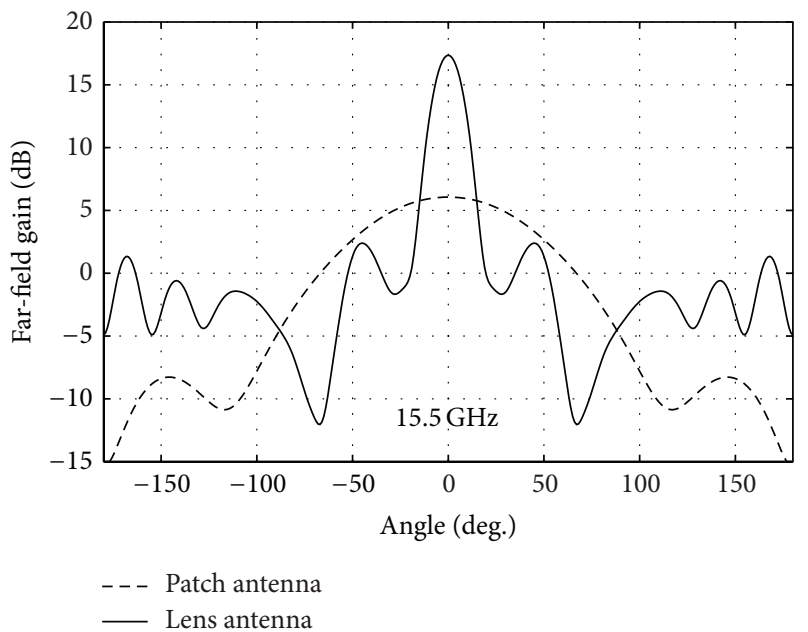

(c)

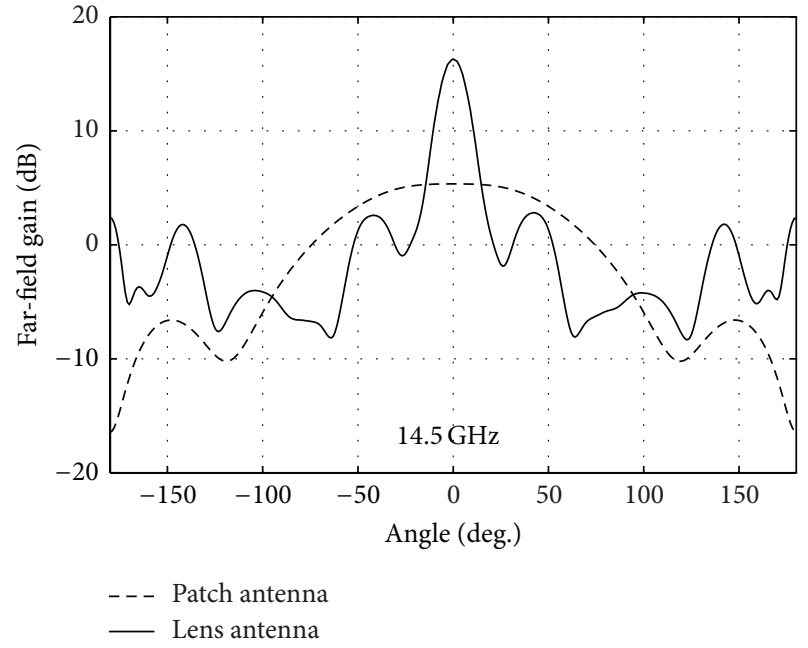

(b)

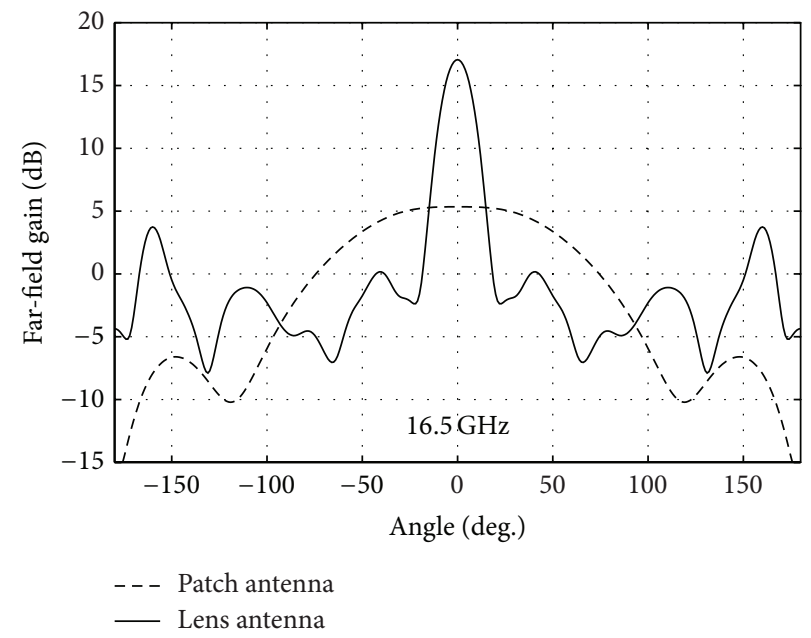

(d)

FIGURE 6: The simulated results of the dielectric metasurface-based lens antenna system. (a) Comparison of $E$-plane far-field radiation pattern of the lens antenna (solid line) and the patch antenna itself (dashed line) at $14.5 \mathrm{GHz}$, (b) at $15.5 \mathrm{GHz}$, and (c) at $16.5 \mathrm{GHz}$. 


\section{Conclusion}

In this paper, we first propose a high-efficiency refractive metasurface composed of electrically thin dielectric blocks, which realize $0 \sim 2 \pi$ phase shift through varying the heights of the building blocks. The full-wave analysis demonstrates that such Huygens' metasurfaces can effectively refract normally incident waves in a wide band. Then, based on the same physical mechanism, a metasurface-based dielectric lens is designed and integrated with a patch antenna to form an ultra-low-profile lens antenna system, which exhibits high directivity, broadband, low loss, and low side-lobe levels. With the fast progress of material technology and 3D printing technology, dielectric Huygens' metasurfaces will find broad applications in microwave, Terahertz, and even optical systems.

\section{Conflict of Interests}

The authors declare that there is no conflict of interests regarding the publication of this paper.

\section{Acknowledgments}

This project is supported by the National Nature Science Foundation of China (Grants nos. 61201030 and 61372045), China Postdoctoral Science Foundation (2012M521048), Collegiate Natural Science Fund of Jiangsu Province (12KJB140010), Natural Science Foundation of Jiangsu Province (Grant no. BK 2012657), and Six Types of Talents Project of Jiangsu Province (2013-XXRJ-010).

\section{References}

[1] J. B. Pendry, D. Schurig, and D. R. Smith, "Controlling electromagnetic fields," Science, vol. 312, no. 5781, pp. 1780-1782, 2006.

[2] J. B. Pendry, A. Aubry, D. R. Smith, and S. A. Maier, "Transformation optics and subwavelength control of light," Science, vol. 337, no. 6094, pp. 549-552, 2012.

[3] S. Guenneau, A. Movchan, G. Pétursson, and S. A. Ramakrishna, "Acoustic metamaterials for sound focusing and confinement," New Journal of Physics, vol. 9, article 399, pp. 1367-2630, 2007.

[4] Y. Li, X. Jiang, R. Li et al., "Experimental realization of full control of reflected waves with subwavelength acoustic metasurfaces," Physical Review Applied, vol. 2, no. 6, Article ID 064002, 2014.

[5] C. L. Holloway, E. F. Kuester, J. A. Gordon, J. O’Hara, J. Booth, and D. R. Smith, "An overview of the theory and applications of metasurfaces: the two-dimensional equivalents of metamaterials," IEEE Antennas and Propagation Magazine, vol. 54, no. 2, pp. 10-35, 2012.

[6] N. F. Yu, P. Genevet, M. A. Kats et al., "Light propagation with phase discontinuities: generalized laws of reflection and refraction," Science, vol. 334, no. 6054, pp. 333-337, 2011.

[7] X.-Y. Jiang, J.-S. Ye, J.-W. He et al., "An ultrathin terahertz lens with axial long focal depth based on metasurfaces," Optics Express, vol. 21, no. 24, pp. 30030-30038, 2013.
[8] S. L. Sun, Q. He, S. Y. Xiao, Q. Xu, X. Li, and L. Zhou, "Gradientindex meta-surfaces as a bridge linking propagating waves and surface waves," Nature Materials, vol. 11, no. 5, pp. 426-431, 2012.

[9] X. Li, S. Xiao, B. Cai, Q. He, T. J. Cui, and L. Zhou, "Flat metasurfaces to focus electromagnetic waves in reflection geometry," Optics Letters, vol. 37, no. 23, pp. 4940-4942, 2012.

[10] X. Chen, L. Huang, H. Mühlenbernd et al., "Dual-polarity plasmonic metalens for visible light," Nature Communications, vol. 3, p. 1198, 2012.

[11] C. Pfeiffer, N. K. Emani, A. M. Shaltout, A. Boltasseva, V. M. Shalaev, and A. Grbic, "Efficient light bending with isotropic metamaterial Huygens' surfaces," Nano Letters, vol. 14, no. 5, pp. 2491-2497, 2014.

[12] Z. B. Wang, J. C. Chen, and M. L. Xue, “Terahertz lenses based on nonuniform metasurfaces," Optics Communications, vol. 338, pp. 585-589, 2015.

[13] M. Moeini-Fard and M. Khalaj-Amirhosseini, "Nonuniform reflect-array antennas," International Journal of $R F$ and Microwave Computer-Aided Engineering, vol. 22, no. 5, pp. 575-580, 2012.

[14] Computer Simulation Technology (CST), Microwave Studio, User's Manual, vol. 4, Computer Simulation Technology (CST), 2013, http://www.cst.com.

[15] P. H. Siegel and R. J. Dengler, "The dielectric-filled parabola: A new millimeter/submillimeter wavelength receiver/transmitter front end," IEEE Transactions on Antennas and Propagation, vol. 39, no. 1, pp. 40-47, 1991.

[16] S. Karimkashi and A. A. Kishk, "Focused microstrip array antenna using a Dolph-Chebyshev near-field design," IEEE Transactions on Antennas and Propagation, vol. 57, no. 12, pp. 3813-3820, 2009.

[17] A. V. Boriskin, A. Rolland, R. Sauleau, and A. I. Nosich, "Assessment of FDTD accuracy in the compact hemielliptic dielectric lens antenna analysis," IEEE Transactions on Antennas and Propagation, vol. 56, no. 3, pp. 758-764, 2008.

[18] X. Wan, W. X. Jiang, H. F. Ma, and T. J. Cui, "A broadband transformation-optics metasurface lens," Applied Physics Letters, vol. 104, no. 15, Article ID 151601, 2014.

[19] A. Demetriadou and Y. Hao, "A grounded slim luneburg lens antenna based on transformation electromagnetics," IEEE Antennas and Wireless Propagation Letters, vol. 10, pp. 15901593, 2011.

[20] B. Zhou, Y. Yang, H. Li, and T. J. Cui, "Beam-steering Vivaldi antenna based on partial Luneburg lens constructed with composite materials," Journal of Applied Physics, vol. 110, no. 8, Article ID 084908, 2011.

[21] A. R. Weily and N. Nikolic, "Dual-polarized planar feed for lowprofile hemispherical Luneburg lens antennas," IEEE Transactions on Antennas and Propagation, vol. 60, no. 1, pp. 402-407, 2012.

[22] E. Hecht, Optics, Addison Wesley, San Francisco, Calif, USA, 4th edition, 2002. 

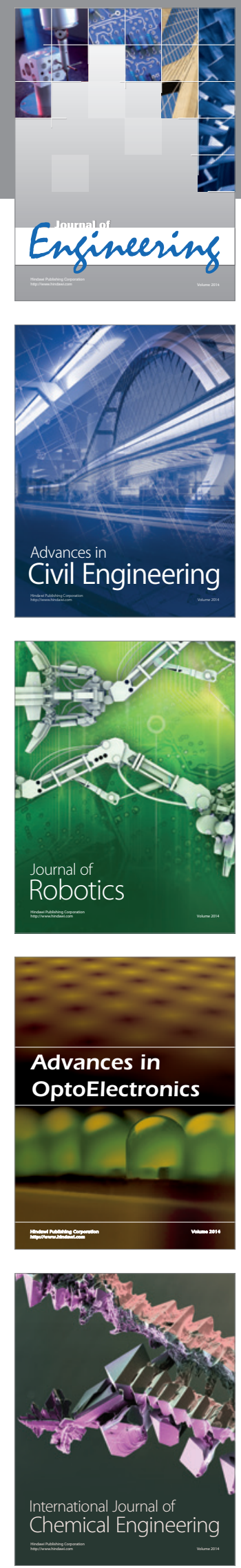

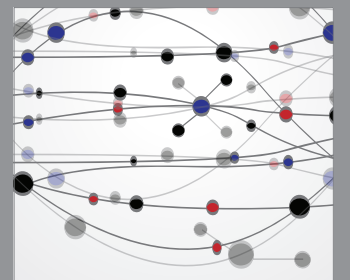

The Scientific World Journal
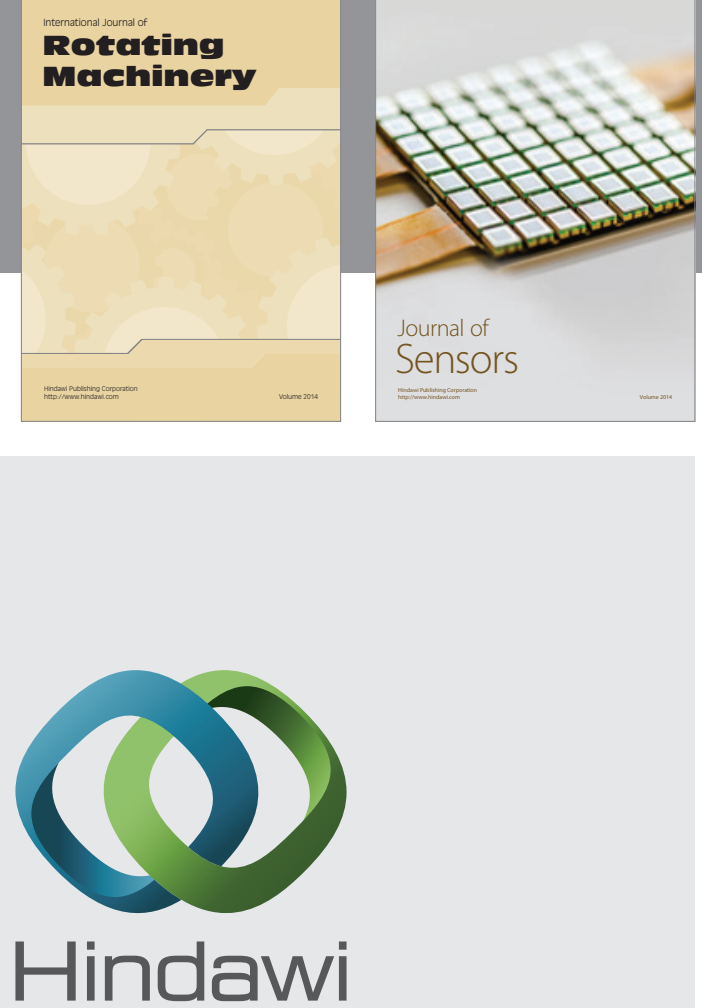

Submit your manuscripts at http://www.hindawi.com
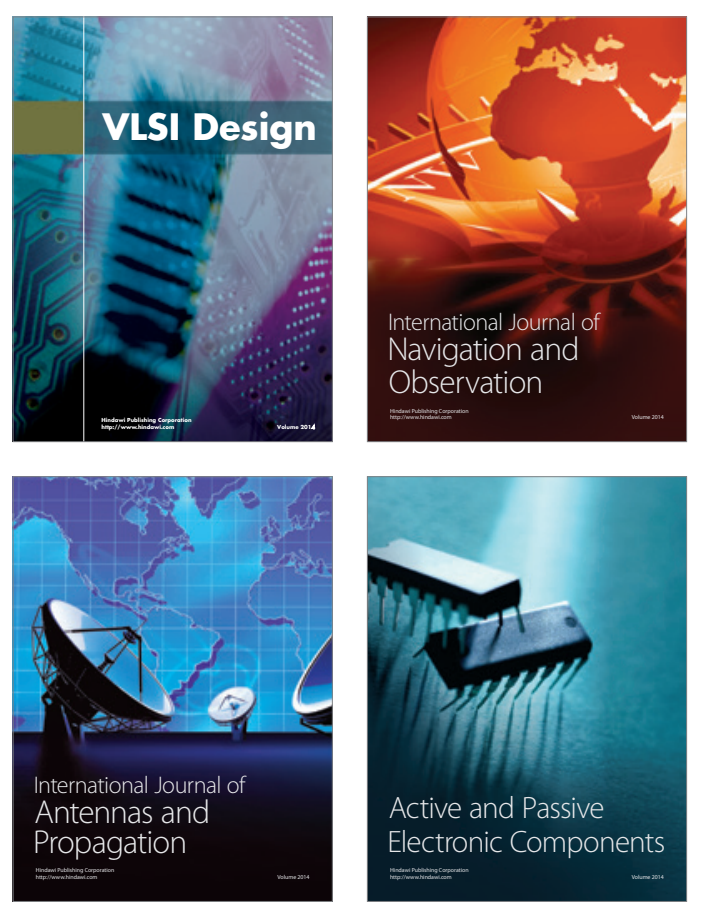
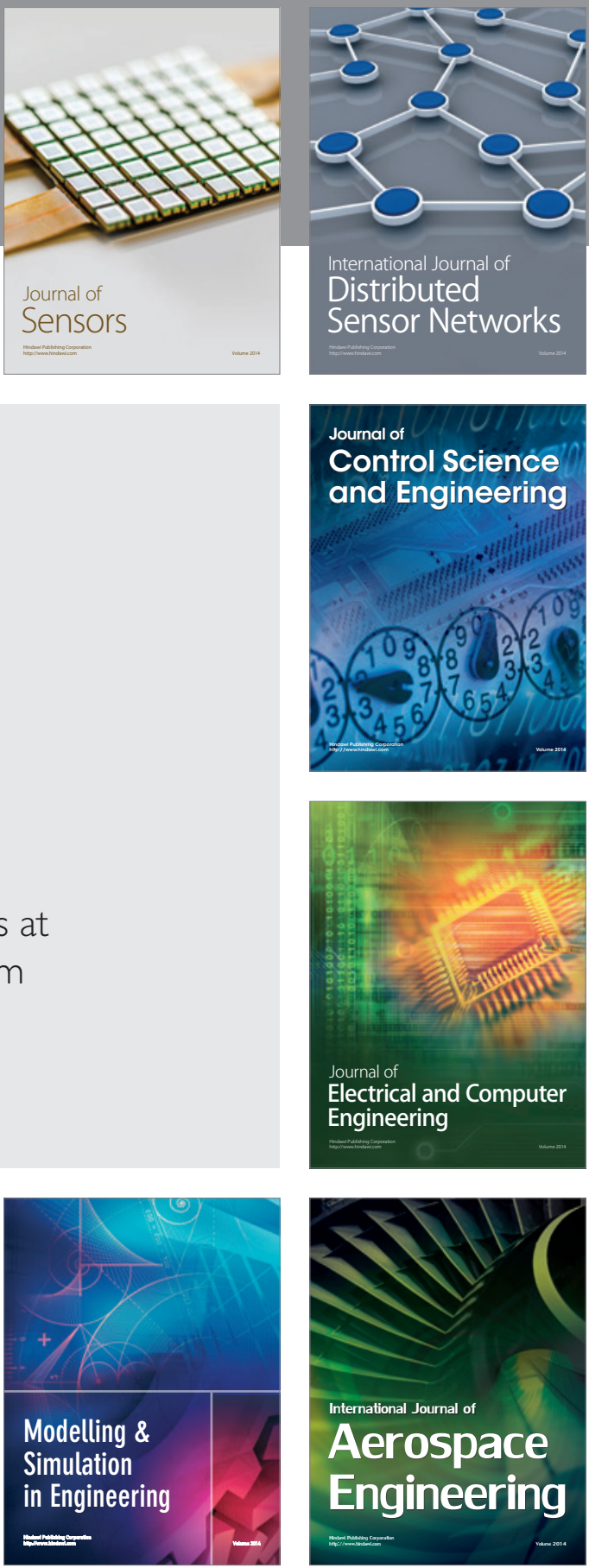

Journal of

Control Science

and Engineering
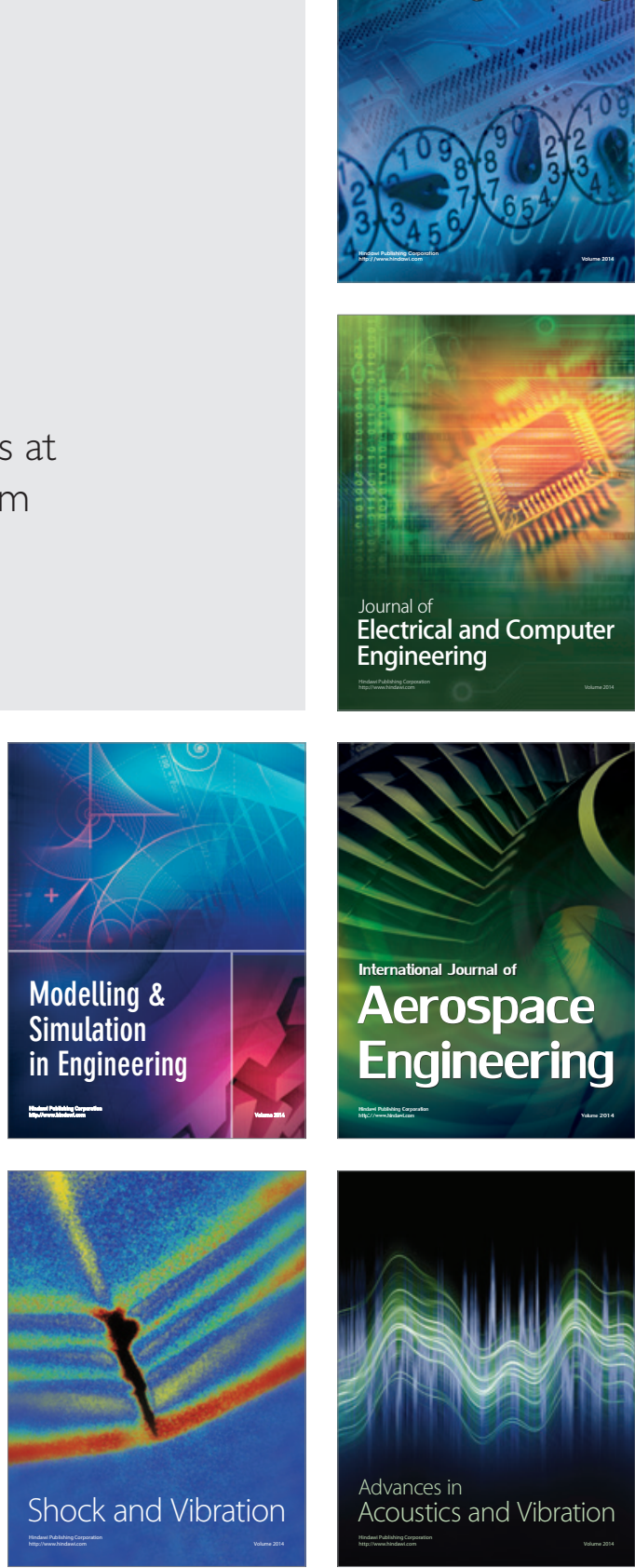